\title{
Electrochemical Sensor Based on Electroactive Polymer and Sulfo-group-functionalized Graphene Oxide Composite for Detection of Dopamine
}

\author{
Ning Li, ${ }^{1}$ Hiroaki Sakamoto, ${ }^{2 *}$ Eiichiro Takamura, ${ }^{2}$ \\ Haitao Zheng, ${ }^{3}$ and Shin-ichiro Suye ${ }^{2}$ \\ ${ }^{1}$ Department of Advanced Interdisciplinary Science and Technology, Graduate School of Engineering, \\ University of Fukui, 3-9-1 Bunkyo, Fukui, Fukui 910-8507, Japan \\ ${ }^{2}$ Department of Frontier Fiber Technology and Science, Graduate School of Engineering, University of Fukui, \\ 3-9-1 Bunkyo, Fukui, Fukui 910-8507, Japan \\ ${ }^{3}$ School of Chemistry and Chemical Engineering, Tiangong University, \\ No. 399 BinShuiXi Road, XiQing District, Tianjin 300387, P.R. China
}

(Received May 20, 2021; accepted July 19, 2021; online published September 6, 2021)

Keywords: electroactive polymerized mediator, functionalized graphene oxide, self-assembly, dopamine

A novel dopamine (DA) sensor based on a polymerized mediator (PEI-Fc) and sulfo-groupfunctionalized graphene oxide (PEI-Fc/SFGO) composite modified electrode was developed. The PEI-Fc/SFGO composite was formed by electrostatic interaction, in which ferrocene $(\mathrm{Fc})$ groups served as an electron transfer mediator to improve the sensitivity. Scanning electron microscopy (SEM) was employed to characterize the surface morphology of the PEI-Fc/SFGO composite. Ultraviolet visible spectroscopy (UV-vis) and Fourier transform infrared (FTIR) spectroscopy were performed to characterize the PEI-Fc and SFGO, respectively. Cyclic voltammetry (CV) was employed to investigate the electrochemical behavior of the PEI-Fc/ SFGO composite modified electrode. Linear sweep voltammetry (LSV) was employed for the quantitative detection of DA. The as-prepared sensor realized a wide linear range of $0.5-100 \mu \mathrm{M}$ with a low limit of detection (LOD) of $0.1 \mu \mathrm{M}$ for the determination of DA. This work provides a simple surface modification technique and a potential electrode material for the detection of DA.

\section{Introduction}

Dopamine (DA), an organic chemical belonging to the catechol amine family, makes a great difference to neurotransmission, emotional expression, and mental activity. ${ }^{(1)}$ However, an abnormal concentration of DA may cause several neurological diseases, for example, Parkinson's disease and schizophrenia are linked to high DA levels. ${ }^{(2)}$ Thus, it is imperative to develop an accurate, rapid, and sensitive monitoring platform for the determination of DA. Owing to the electroactivity of DA, ${ }^{(3)}$ electrochemical sensors have recently opened up new avenues for the determination of DA. However, ascorbic acid (AA) and uric acid (UA) co-exist with DA in real biological samples. ${ }^{(4)}$ AA and UA are not only electroactive molecules, but they also exhibit

*Corresponding author: e-mail: hi-saka@u-fukui.ac.jp https://doi.org/10.18494/SAM.2021.3435 
similar oxidation potentials to DA, ${ }^{(4,5)}$ so DA sensors with low sensitivity and poor selectivity will induce the electrooxidation of DA, UA, and AA at the same oxidation potential, causing the inaccurate detection of the DA concentration. Therefore, for the practical application of DA sensors, high sensitivity and excellent selectivity are especially important. To improve the sensitivity and selectivity, different types of material, such as graphene-based materials with excellent conductivity and large surface area, ${ }^{(6,7)}$ were introduced onto the surface of conventional electrodes [gold, platinum, or glassy carbon (GC)]. These materials significantly increased the electroanalytical ability for DA, perhaps driven by the enhanced heterogeneous electron transfer kinetics, ${ }^{(6)}$ high specific surface area, ${ }^{(8)}$ and excellent conductivity, enabling the oxidation of DA, UA, and AA at different potentials. ${ }^{(9)}$

Graphene oxide (GO), one of the derivatives of graphene, is usually used to improve the performance of electrochemical sensors because of its excellent properties such as large surface area. ${ }^{(10)}$ However, the poor dispersion of GO is still the main cause of the detachment of the GO film from the electrode surface, which in turn can reduce the sensitivity, reproducibility, repeatability, and stability of electrochemical sensors. However, GO has a considerable number of oxygen-containing functional groups, which make further chemical functionalization of GO much easier. ${ }^{(11)}$ The functionalization of GO is a good approach to obtaining well-dispersed GO. ${ }^{(12)}$ Modifying the surface of GO with appropriate hydrophilic functional groups not only improves its dispersibility but also expands its range of applications. For example, sulfo-groups have strong hydrophilic properties, and functionalizing GO with sulfonic groups greatly improves its hydrophilicity. ${ }^{(13)}$ Although the problem of poor dispersion can be solved by introducing strong hydrophilic functional groups on GO, the conductivity of GO is still unsatisfactory because of the large number of oxygen-containing functional groups, ${ }^{(14)}$ leading to insufficient sensitivity. Therefore, it is very important to further improve the sensitivity of electrodes modified with functionalized GO.

Ferrocene $(\mathrm{Fc})$ and its derivatives are often utilized in electrochemical sensors owing to their high redox activity, generation of comparatively stable redox forms, and optimum electrochemical reversibility. ${ }^{(15,16)}$ The $\mathrm{Fc}^{+} / \mathrm{Fc}$ couple behaves as a redox mediator towards various analytes and significantly promotes the electrochemical response. ${ }^{(15)}$ Nevertheless, if Fc is directly modified on the electrode surface, it simply dissolves in the bulk solution, which will result in the diffusion and loss of $\mathrm{Fc}$ from the electrode surface. ${ }^{(17)}$ Therefore, it is important to find how to reduce the loss of Fc when modified on an electrode surface. This issue can be addressed by bonding Fc with polymer chains. ${ }^{(18)}$ Therefore, the development of Fc-bonded polyethylenimine (PEI-Fc), a polymerized mediator, was first proposed in our previous paper. ${ }^{(19)}$ The introduction of a PEI polymer backbone not only prevented the Fc mediator from diffusion but also introduced positive charges donated by amino groups. In our previous work, ${ }^{(20)}$ a DA sensor based on PEI-Fc/sulfo-group-functionalized graphene oxide (SFGO) multilayer film modification was fabricated through a layer-by-layer (LBL) assembly method. However, the LBL assembly method is very time-consuming and uses materials inefficiently. In other studies, carbon nanotubes (CNTs) and metals such as gold nanoparticles were used to improve the sensitivity of DA sensors, ${ }^{(21,22)}$ although the electrode materials were very expensive and the preparation process was complex. 
In this study, a novel sensor for the detection of DA consisting of a PEI-Fc/SFGO composite as an electrode material was developed, in which the $\mathrm{Fc}^{+} / \mathrm{Fc}$ couple is a good mediator to significantly enhance the electrochemical response toward the electrooxidation of DA. Moreover, the prepared SFGO disperses homogeneously and has large surface area and high mechanical stability, which improve the mediator loading and enable tight assembly with PEI-Fc, preventing the leakage of PEI-Fc. We thus propose a low-cost and easily fabricated material with potential application in DA sensors.

\section{Experimental Procedure}

\subsection{Chemicals and materials}

GO in powder form and ferrocene carboxaldehyde $\left(\mathrm{C}_{11} \mathrm{H}_{10} \mathrm{FeO}\right)$ were purchased from Tokyo Chemical Industry, Japan. Ethyleneimine (polymer) (PEI, 30\% in water, M.W. 60000-80000) and DA hydrochloride (DA, M.W. 189.64) were obtained from Nacalai Tesque, Inc., Kyoto, Japan. UA, L-AA, and D(+)-glucose were all bought from Wako Pure Chemical Industries, Ltd., Osaka, Japan. All chemicals used here were of analytical grade and all aqueous solutions were prepared with reverse osmosis $(\mathrm{RO})$ water.

\subsection{Methods and apparatus}

Ultraviolet-visible spectroscopy (UV-vis, GENESYS 10S) was employed to characterize the prepared electroactive polymerized mediator. Fourier transform infrared spectroscopy (FTIR; LabRAM HR-800-Horiba) was performed to analyze the functional groups of the as-prepared materials. The surface morphology of the modified electrodes was observed using a scanning electron microscopy (SEM) system (JEOL, Japan) at an acceleration voltage of $15 \mathrm{kV}$. The samples used for SEM observation were prepared by adding a suspension of the prepared materials dropwise on the surface of a glassy carbon (GC) sheet, which was followed by drying in air at room temperature.

Electrochemical measurements were performed on a CHI 660E electrochemical workstation. The electrochemical behavior of different modified electrodes was investigated by cyclic voltammetry (CV), and linear sweep voltammetry (LSV) was used for the detection of DA. A typical three-electrode system was used, with a bare glassy carbon electrode (GCE) $3.0 \mathrm{~mm}$ diameter, $0.071 \mathrm{~cm}^{2}$, BAS Co., Ltd., Osaka, Japan) or a modified GCE as the working electrode and $\mathrm{Pt}$ wire and $\mathrm{Ag} / \mathrm{AgCl}$ electrodes serving as the counter and reference electrodes, respectively. All electrochemical measurements were carried out at room temperature.

\subsection{Preparation of PEI-Fc}

The combination of high-molecular-weight polymer and electroactive ferrocene groups was synthesized as in our previous work with slight modification. ${ }^{(19)}$ Firstly, $0.039 \mathrm{~g}$ ferrocene carboxaldehyde (Fc-CHO) dissolved in $3 \mathrm{~mL}$ ethanol solution was slowly added to the same 
volume of ethanol solution containing $0.3 \mathrm{~g}$ PEI ( $30 \%$ in water) with continuous stirring for $1 \mathrm{~h}$ at room temperature. Then, $0.008 \mathrm{~g} \mathrm{NaBH}_{4}$ was dissolved in the mixture, which was subsequently stirred for $1 \mathrm{~h}$. The obtained solution was purified by membrane dialysis against distilled water for $36 \mathrm{~h}$. The obtained solution after dialysis is referred to as PEI-Fc solution. Finally, the PEI-Fc solution was diluted to $5 \mathrm{mg} \mathrm{mL}^{-1}$ using RO water and $\mathrm{pH}$ was adjusted to 7.0.

\subsection{Synthesis of PEI-Fc/SFGO composite}

The SFGO was prepared in accordance with a previous study with slight modification. ${ }^{(23)}$ Briefly, sulfo-groups $\left(-\mathrm{SO}_{3} \mathrm{H}\right)$ were introduced to the surface of $\mathrm{GO}$ by the diazonium salt coupling method. First, $25 \mathrm{mg}$ sulfanilic acid, $6 \mathrm{~mL}$ ice $\mathrm{HCl}$ solution $\left(v_{\text {ice RO water }} / v_{\mathrm{HCl}}\right.$ (concentrated grade): 5:1), and $0.1 \mathrm{~g} \mathrm{NaNO}_{2}$ powder were mixed in $10 \mathrm{~mL} \mathrm{NaOH}$ solution (2 wt\%) under mechanical stirring for $15 \mathrm{~min}$ at $0{ }^{\circ} \mathrm{C}$. Then, the mixture was poured into $50 \mathrm{~mL}$ GO suspension $\left(1 \mathrm{mg} \mathrm{mL} \mathrm{m}^{-1}\right.$ ), which was followed by continuous mechanical stirring for $4 \mathrm{~h}$ at $0{ }^{\circ} \mathrm{C}$. Finally, the product was washed by centrifugation using distilled water until its $\mathrm{pH}$ became 7.0, then dried at room temperature. After the preparation of PEI-Fc and SFGO, the PEI-Fc/SFGO composite was prepared by mixing $100 \mu \mathrm{L}$ PEI-Fc solution $\left(5 \mathrm{mg} \mathrm{mL}^{-1}\right)$ with $500 \mu \mathrm{L}$ SFGO dispersion (5 mg mL ${ }^{-1}$ in $\mathrm{RO}$ water), which was followed by ultrasonication for $40 \mathrm{~min}$.

\subsection{Fabrication of PEI-Fc/SFGO modified electrode}

Before the fabrication of the PEI-Fc/SFGO composite modified electrode, GCE was first polished with 50 and $0.05 \mu \mathrm{m}$ alumina/water slurries until the surface had a mirror finish. After rinsing in distilled water, the polished electrode was cleaned by mild ultrasonication with distilled water for about $1 \mathrm{~min}$. Then, $5 \mu \mathrm{L}$ aqueous dispersion of the as-synthesized PEI-Fc/ SFGO composite was added dropwise on the surface of the pre-prepared GCE and was subsequently dried at room temperature in air to obtain PEI-Fc/SFGO/GCE. Next, the PEI-Fc/ SFGO composite modified electrode was electrochemically treated by $\mathrm{CV}$ for 15 successive cycles in $0.1 \mathrm{M}$ phosphate buffer solution (PBS) ( $\mathrm{pH} 7.0$, containing $0.1 \mathrm{M} \mathrm{KCl}$ ) in the potential range of 0.7 to $-1.1 \mathrm{~V}$ vs $\mathrm{Ag} / \mathrm{AgCl}$ with a scan rate of $100 \mathrm{mV} \mathrm{s}^{-1}$ to weaken the background current from the $\mathrm{Fc}^{+} / \mathrm{Fc}$ couple. Finally, the PEI-Fc/SFGO/GCE was thoroughly rinsed with RO water five times to remove loose Fc groups. The fabrication process is shown in Fig. 1. The asprepared electrode was kept in a PBS buffer at room temperature until further use.

\section{Results and Discussion}

\subsection{Analytical characterization}

First, the as-prepared PEI-Fc was characterized by UV-vis. No peaks can be seen in the UVvis spectrum of PEI in Fig. 2 because there are no unsaturated groups in PEI. In the UV-vis spectrum of Fc-CHO, two strong peaks at 230 and $275 \mathrm{~nm}$ can be clearly observed, which 


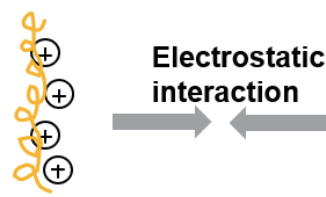

PEI-FC

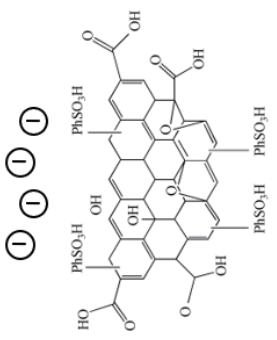

SFGO

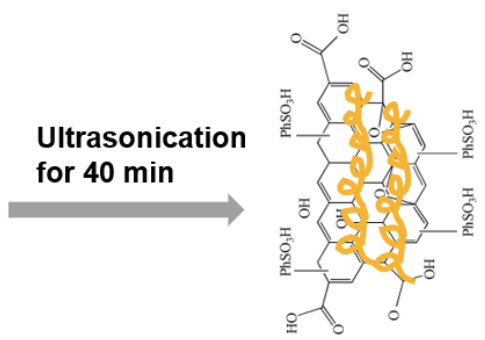

PEI-Fc/SFGO composite
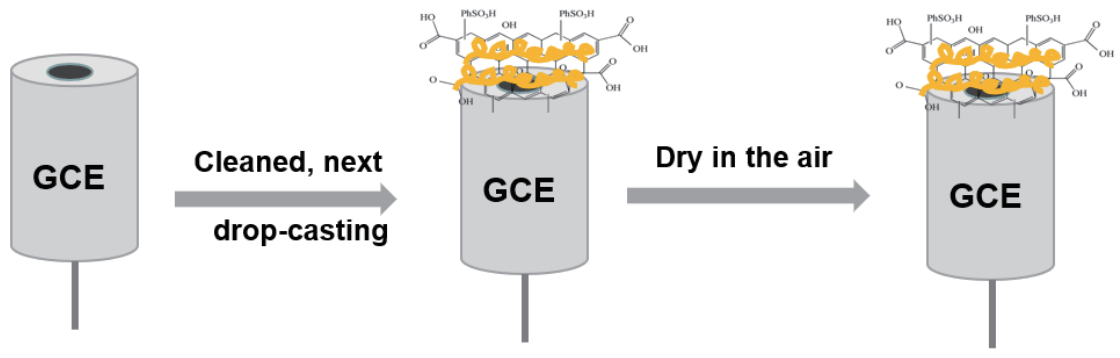

PEI-Fc/SFGO/GCE

Fig. 1. (Color online) Fabrication process of PEI-Fc/SFGO/GCE.

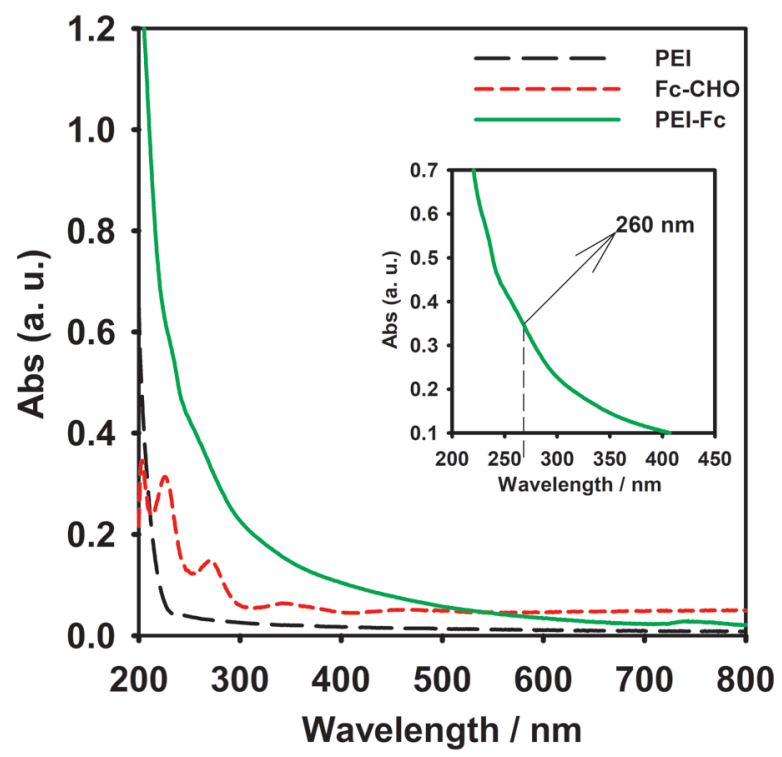

Fig. 2. (Color online) UV-vis spectra of PEI, Fc-CHO, and PEI-Fc. Inset: enlarged view of UV-vis spectrum of PEI-Fc.

correspond to the - $\mathrm{CHO}$ groups and benzene ring structure, respectively. In the UV-vis spectrum of PEI-Fc, the typical strong peak of the -CHO groups at $230 \mathrm{~nm}$ almost disappeared, which was attributed to the reaction between the -CHO and amino groups. On the other hand, the typical benzene ring peak exhibited a blueshift to $260 \mathrm{~nm}$, which is ascribed to the introduction of the PEI backbone into the benzene ring structure, confirming the successful formation of PEI-Fc. 
After confirming the successful preparation of PEI-Fc, the GO and synthesized SFGO were analyzed by FTIR, as shown in Fig. 3. In the FTIR spectrum of GO, two typical strong peaks appeared at 1720 and $1610 \mathrm{~cm}^{-1}$, corresponding to the stretching of carboxyl groups and the $\mathrm{C}=\mathrm{C}$ stretching of the aromatic ring, respectively. ${ }^{(24)}$ In addition, very clear peaks of C-O and C-O-C were located at 1168 and $1050 \mathrm{~cm}^{-1}$, (25) respectively, illustrating the existence of a large number of oxygen functional groups on the surface of GO. For the SFGO FTIR spectrum, the classic $\mathrm{C}=\mathrm{C}$ stretching of the aromatic ring can still be clearly observed at $1610 \mathrm{~cm}^{-1}$. Note that the $\mathrm{C}=\mathrm{O}$ stretching of carboxyl groups was markedly weakened, as shown in the FTIR spectrum of SFGO. This phenomenon was probably caused by the addition of $\mathrm{NaNO}_{2}$ during the preparation of functionalized GO, which reduced various carboxyl groups. Moreover, the SFGO spectrum also exhibited the characteristic peaks of sulfo-groups $\left(-\mathrm{SO}_{3} \mathrm{H}\right)$ at $1250,1112,1014$, and $850 \mathrm{~cm}^{-1}$, confirming the successful introduction of sulfo-groups. ${ }^{(13,25)}$

\subsection{Surface morphological characterization}

The surface morphologies of bare/GCE, SFGO/GCE, and PEI-Fc/SFGO/GCE were observed by SEM. It can be seen from Fig. 4(a) that the bare GCE had a very smooth surface. The SFGO in SFGO/GCE comprised small circular flakes that were spread uniformly on the electrode [Fig. 4(b)]. For the PEI-Fc/SFGO composite surface morphology [Fig. 4(c)], the SFGO flakes were less apparent. This is probably because the aggregation of the PEI polymer on the SFGO surface rendered the SFGO surface almost invisible.

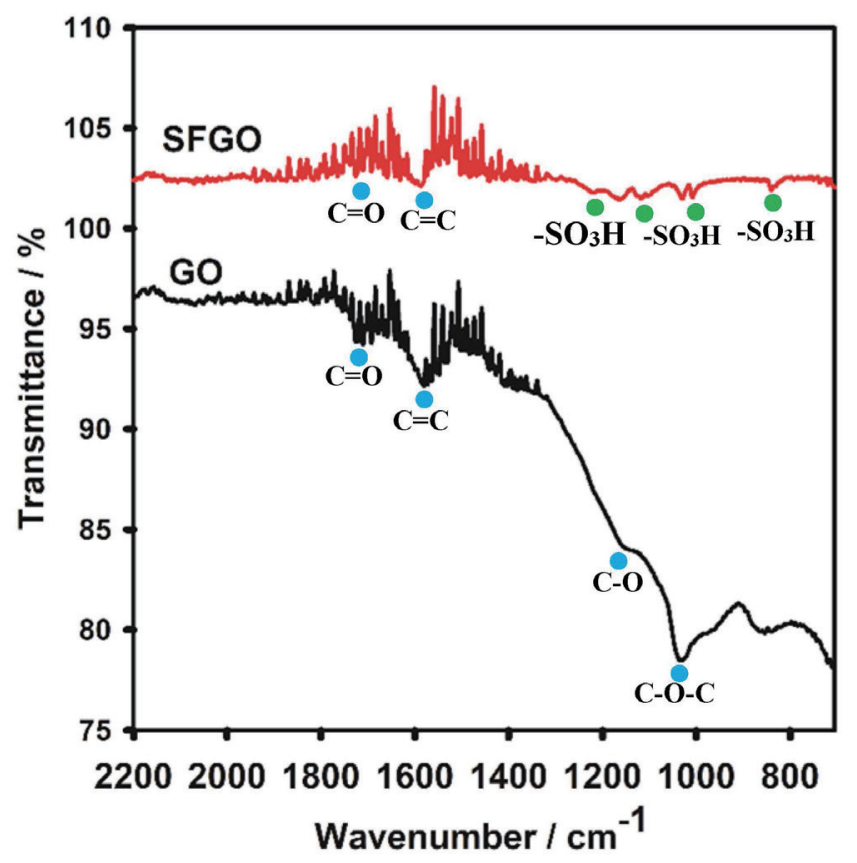

Fig. 3. (Color online) FTIR spectra of GO and SFGO. 


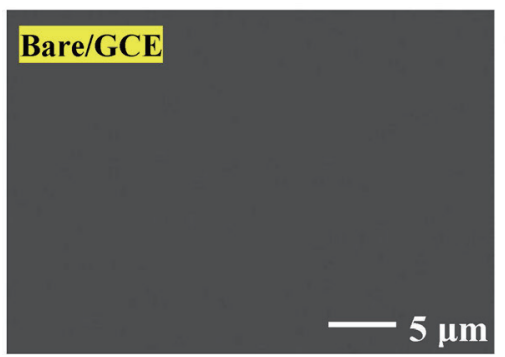

(a)

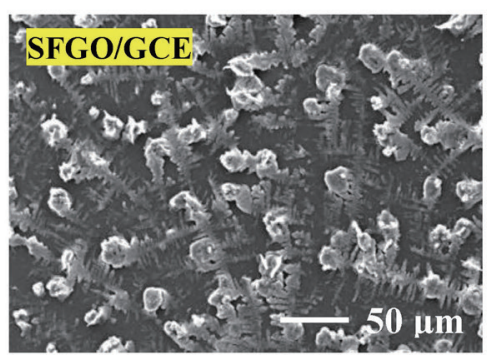

(b)

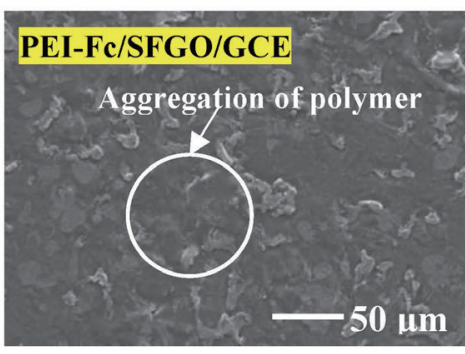

(c)

Fig. 4. (Color online) SEM images of (a) bare/GCE, (b) SFGO/GCE, and (c) PEI-Fc/SFGO/GCE.

\subsection{Electrochemical characterization}

$\mathrm{CV}$ was carried out to study the electrochemical behavior of PEI-Fc/SFGO/GCE in $0.1 \mathrm{M}$ PBS (pH 7.0, containing $0.1 \mathrm{M} \mathrm{KCl}$ ) in the absence of DA at a scan rate of $20 \mathrm{mV} \mathrm{s}^{-1}$. The resulting CV graph is shown in Fig. 5(a). In the absence of DA, a pair of reversible redox peaks was displayed for PEI-Fc/SFGO/GCE, which is due to the electroactive property of the $\mathrm{Fc}^{+} / \mathrm{Fc}$ couple. This couple was electrochemically oxidized at $390 \mathrm{mV}$ and reduced at $310 \mathrm{mV}$ with a peak-to-peak separation $(\triangle E p)$ of $80 \mathrm{mV}$, demonstrating that the $\mathrm{Fc}^{+} / \mathrm{Fc}$ couple exhibited reversible redox behavior on the electrode surface. Figure 5(b) shows the CV responses of bare/ GCE, PEI/SFGO/GCE, PEI-Fc/GO/GCE, and PEI-Fc/SFGO/GCE in $0.1 \mathrm{M}$ PBS in the presence of $500 \mu \mathrm{M} \mathrm{DA}$ at $20 \mathrm{mV} \mathrm{s}^{-1}$. A pair of tiny redox peaks with a wide $\Delta E p$ was observed for bare/ GCE $(\triangle E p: 184 \mathrm{mV})$ and PEI/SFGO/GCE $(\triangle E p: 120 \mathrm{mV})$, illustrating the quasi-reversible and sluggish electrochemical reaction process of DA. However, for PEI-Fc/GO/GCE, only a clear and wide anodic peak at $365 \mathrm{mV}$ appeared, indicating the irreversible reaction process of DA. Considering the surface morphology of $\mathrm{GO} / \mathrm{GCE}$, this result is probably due to the aggregation of GO on the electrode surface, which can reduce its conductivity and electron transfer rate. An extremely sharp anodic peak with the highest current response at the lowest potential of $178 \mathrm{mV}$ was observed at PEI-Fc/SFGO/GCE, demonstrating faster electron transfer kinetics and a larger electroactive surface area than all the other modified electrodes (bare/GCE, PEI/SFGO/GCE and $\mathrm{PEI}-\mathrm{Fc} / \mathrm{GO} / \mathrm{GCE})$.

It was shown that PEI-Fc/SFGO/GCE exhibited the largest anodic peak current $\left(I_{p a} \sim 8.5 \mu \mathrm{A}\right)$ corresponding to the electrooxidation of DA, which is around eight times higher than those of bare/GCE and PEI/SFGO/GCE, and about 1.7 times higher than that of PEI-Fc/GO/GCE. The dramatic boost in the anodic peak current and the oxidation potential lower than those of bare/ GCE and PEI/SFGO/GCE provide convincing evidence that the $\mathrm{Fc}^{+} / \mathrm{Fc}$ couple can facilitate the electrooxidation of DA as a mediator. In addition, in the presence of DA, the anodic peak current increased greatly, while the cathodic peak current decreased markedly compared with those for $\mathrm{PEI}-\mathrm{Fc} / \mathrm{SFGO} / \mathrm{GCE}$ in the absence of DA. This result is in agreement with the work performed by Diao's group, ${ }^{(26)}$ who clearly gave the reason why the cathodic peak current decreased. ${ }^{(26)}$

Moreover, the peak current response at PEI-Fc/SFGO/GCE that is higher than that at PEI-Fc/ $\mathrm{GO} / \mathrm{GCE}$ reveals that the sulfo-group-functionalized $\mathrm{GO}$ is able to improve the electrochemical 


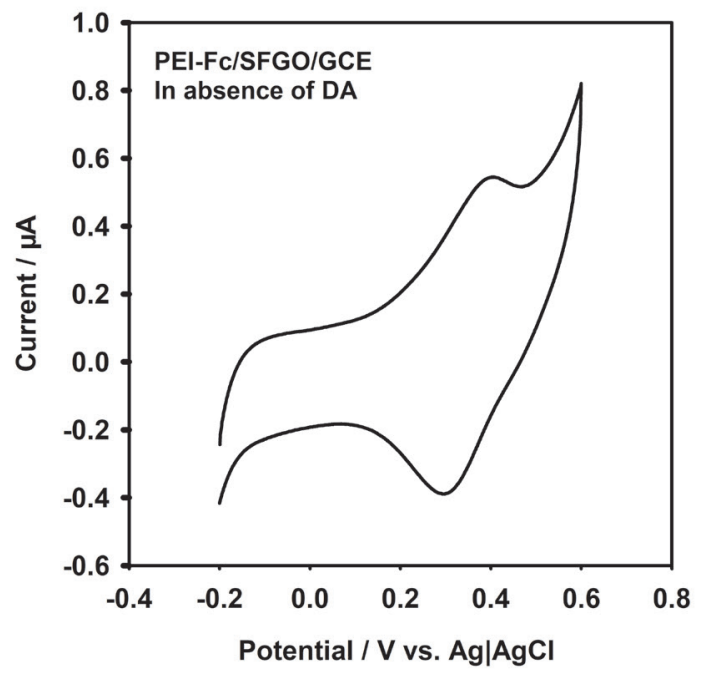

(a)

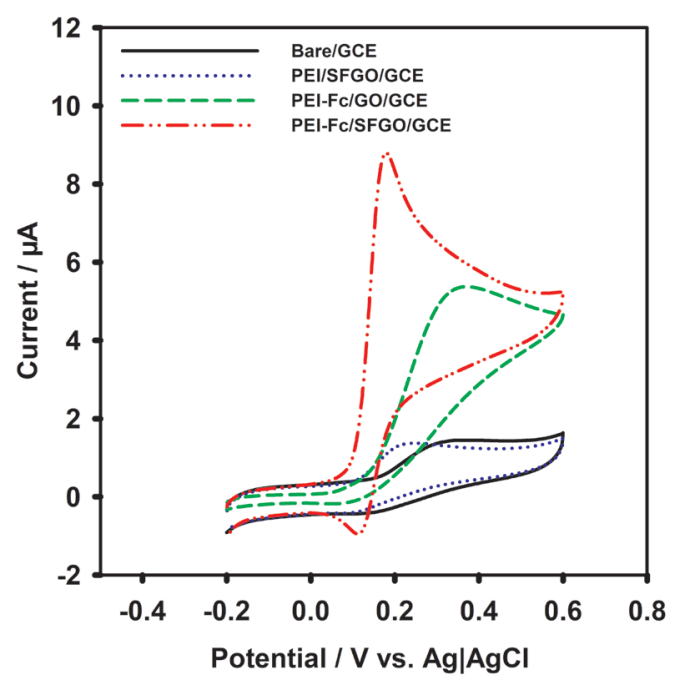

(b)

Fig. 5. (Color online) (a) $\mathrm{CV}$ responses recorded at $\mathrm{PEI}-\mathrm{Fc} / \mathrm{SFGO} / \mathrm{GCE}$ in $0.1 \mathrm{M} \mathrm{PBS}$ (pH 7.0, containing $0.1 \mathrm{M} \mathrm{KCl}$ ) in the absence of DA and (b) $\mathrm{CV}$ responses recorded at various modified electrodes in $0.1 \mathrm{M}$ PBS in the presence of $500 \mu \mathrm{M}$ DA at $20 \mathrm{mV} \mathrm{s}^{-1}$.

performance of GO. This phenomenon can be explained by the sulfo-groups greatly improving the hydrophilicity and dispersity of GO, which can protect the GO from aggregation and enhance the electron transfer of the electrode. Alternatively, according to the FTIR spectrum of SFGO, it is also possible that during the functionalization of $\mathrm{GO}$, a number of carboxyl groups were reduced. The reduction of oxygen-containing functionalized groups can also improve the conductivity of GO. ${ }^{(27)}$

\subsection{Investigation of electrochemical reaction process}

To study the effect of the scan rate, the CV responses of the PEI-Fc/SFGO composite modified electrode at different scan rates from 5 to $125 \mathrm{mV} \mathrm{s}^{-1}$ in $0.1 \mathrm{M}$ PBS containing $500 \mu \mathrm{M}$ DA were investigated. As shown in Fig. 6(a), it is clear that the anodic and cathodic peak currents increased with the scan rate. In addition, it can be seen in Fig. 6(a) that when the scan rate surpassed $40 \mathrm{mV} \mathrm{s}^{-1}, \Delta E p$ increased monotonically. That means that the redox reaction process tends to be less reversible with increasing scan rate, indicating that the electrode reaction process is quasi-reversible. It is shown in Fig. 6(b) that both types of peak current had a good linear relationship with the square root of the scan rate, suggesting that the reaction process is diffusion-controlled. $^{(2)}$ Also, when the scan rate is higher than $40 \mathrm{mV} \mathrm{s}^{-1}$, the diffusion rate is lower than the scan rate, resulting in the increased irreversibility of the reaction.

To study the effect of $\mathrm{pH}$ on the electrooxidation of DA at PEI-Fc/SFGO/GCE, the $\mathrm{pH}$ of PBS was varied from 5.0 to 9.0. It is shown in Fig. 7(a) that the anodic peak potentials underwent a negative shift with increasing $\mathrm{pH}$, confirming that electron transfer is involved in the oxidation of DA. Moreover, Fig. 7(b) shows that the anodic peak potential had a linear relationship with $\mathrm{pH}$ 


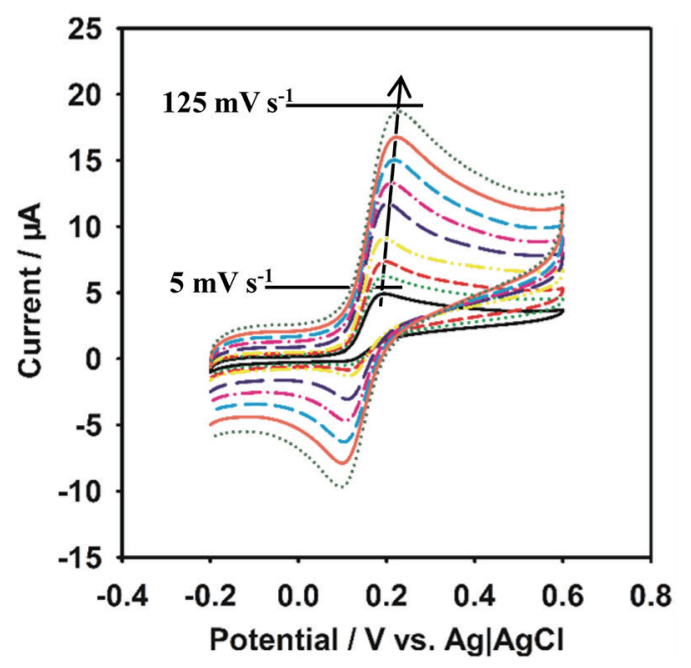

(a)

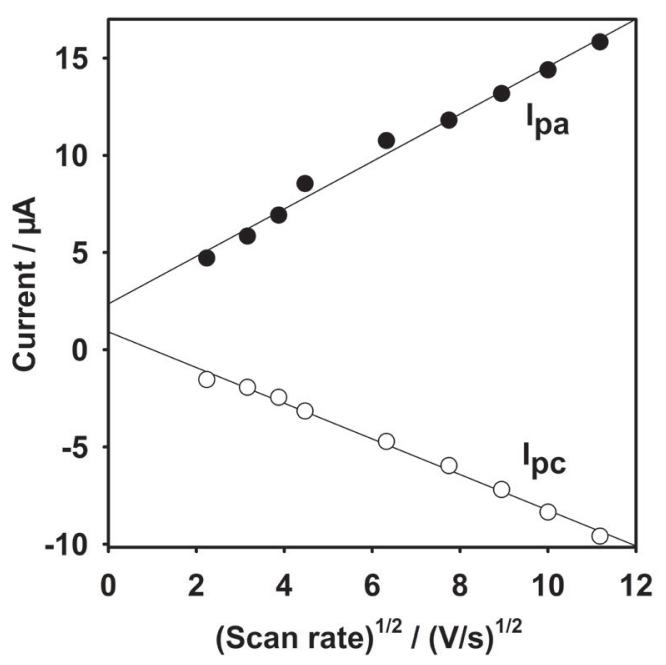

(b)

Fig. 6. (Color online) (a) CV responses recorded at PEI-Fc/SFGO/GCE in $0.1 \mathrm{M}$ PBS (pH 7.0, containing $0.1 \mathrm{M}$ $\mathrm{KCl})$ in the presence of $500 \mu \mathrm{M}$ DA at various scan rates $\left(5,10,15,20,40,60,80,100\right.$, and $\left.125 \mathrm{mV} \mathrm{s}^{-1}\right)$ and (b) corresponding redox peak currents as a function of square root of scan rate.

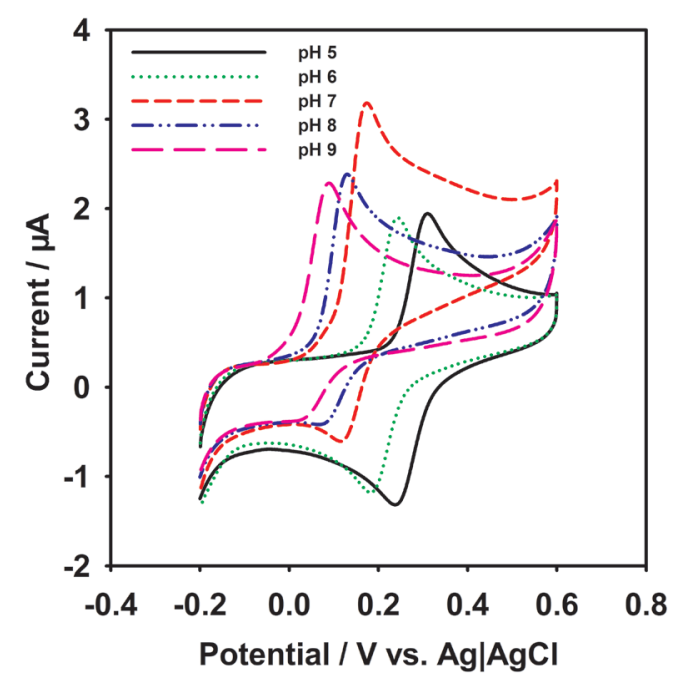

(a)

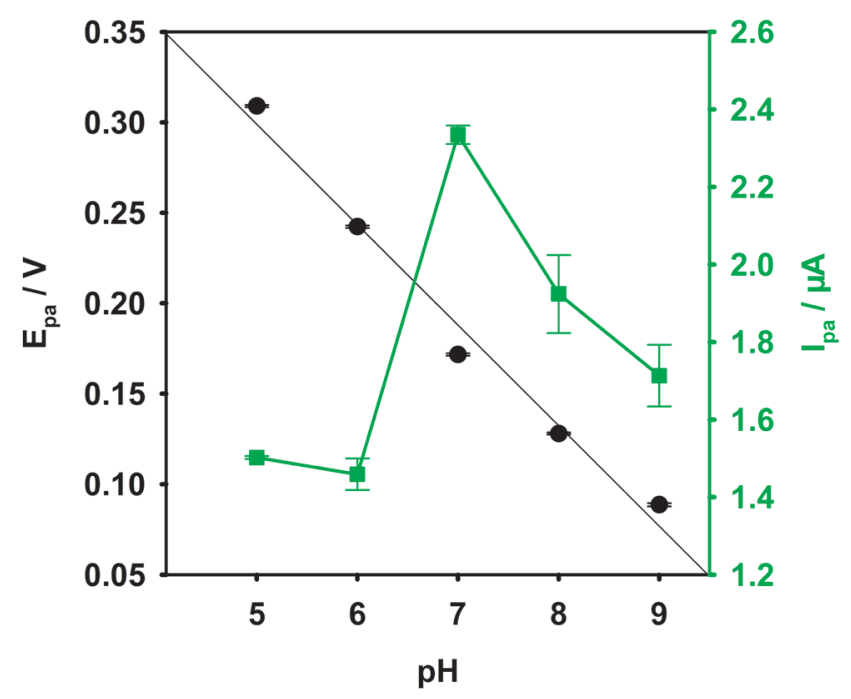

(b)

Fig. 7. (Color online) (a) CV responses recorded at PEI-Fc/SFGO/GCE in $0.1 \mathrm{M}$ PBS (containing $0.1 \mathrm{M} \mathrm{KCl}$ and $100 \mu \mathrm{M}$ DA) with different $\mathrm{pH}$ values $(\mathrm{pH} 5.0-9.0)$ and (b) corresponding anodic peak potentials $\left(E_{p a}\right)$ and anodic peak currents $\left(I_{p a}\right)$ as a function of $\mathrm{pH}$. Error bars show the standard error of the mean of three groups of data from three independent electrodes.

with the linear regression equation $E_{p a}=0.3552-0.0554 \mathrm{pH}\left(R^{2}=0.9833\right)$. The calculated slope $(55 \mathrm{mV} / \mathrm{pH})$ of the linear regression equation is near the Nernst value $(59 \mathrm{mV} / \mathrm{pH})$, which means that two protons and two electrons are involved in the redox process of DA. ${ }^{(28)}$ It is also shown in Fig. 7(b) that the anodic peak sensitivity was highest at $\mathrm{pH}$ 7. Thus, $\mathrm{pH} 7$ was selected for further detection of DA. 


\subsection{Investigation of application for DA detection}

\subsubsection{Reaction principle of DA at PEI-Fc/SFGO/GCE}

The reaction principle of DA at the PEI-Fc/SFGO composite modified electrode is shown in Fig. 8. The electroactive DA molecules are oxidized to DA ortho-quinone (DAQ) as a by-product and produce two electrons. Simultaneously, $\mathrm{Fc}^{+}$is reduced by one of the produced electrons, then the mediator $\mathrm{Fc}$ is re-oxidized on the electrode surface while simultaneously transferring the electron to the electrode surface. Every electron transferred to the electrode surface by the mediator corresponds to the oxidation of a DA molecule. Therefore, it is possible to detect the concentration of DA in the solution by measuring the anodic current response.

\subsubsection{Continuous detection of DA by LSV}

LSV was performed for the continuous detection of DA by using PEI-Fc/SFGO/GCE. Figure 9(a) shows the LSV anodic current response to the successive addition of DA in $0.1 \mathrm{M}$ PBS from -0.1 to $0.5 \mathrm{~V}$ at a scan rate of $20 \mathrm{mV} \mathrm{s}^{-1}$. It is shown that the PEI-Fc/SFGO composite can oxidize DA at a potential of $0.18 \mathrm{~V}$, which is significantly lower than the value presented by Diao's group. ${ }^{(26)}$ In addition, the anodic peak current response increased monotonically with each successive addition of DA. Furthermore, as shown in Fig. 9(b), an extremely strong linear relationship between the anodic peak current and the DA concentration was obtained in the range of $0.5-100 \mu \mathrm{M}$. The linear regression equation is $I_{p a}=0.0359+0.0171 C_{D A}(\mu \mathrm{M})\left(R^{2}=\right.$ 0.9991). This linear range is wider than that of the DA sensor based on $\mathrm{Fc}$ as an electron transfer mediator in a previous work. ${ }^{(29)}$ The limit of detection (LOD) was estimated to be $0.1 \mu \mathrm{M}$ at a signal-to-noise $(\mathrm{S} / \mathrm{N})$ value of 3, which is lower than that of a previously reported DA sensor based on Fc as an electron transfer mediator. ${ }^{(26)}$ A comparison of the as-prepared DA sensor in the present work with those in recent studies is shown in Table 1. It can be seen that the DA sensor based on PEI-Fc/SFGO fabricated in the present work has a wider linear range than the

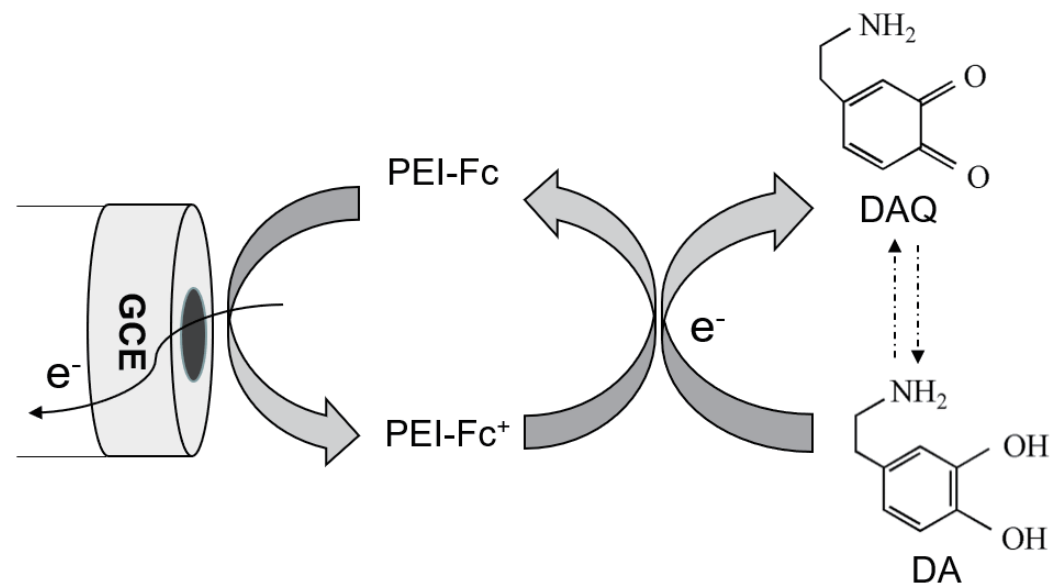

Fig. 8. Illustration of the reaction principle of DA at the as-prepared electrode. 


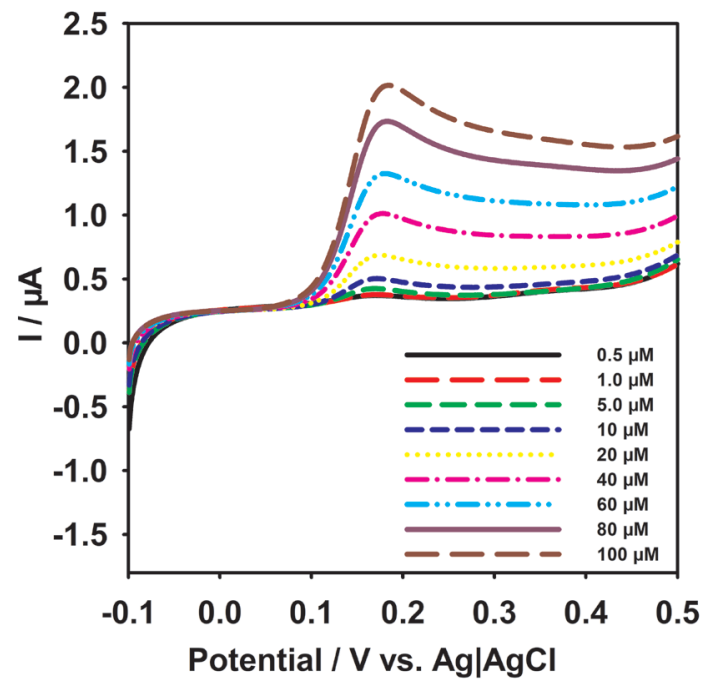

(a)

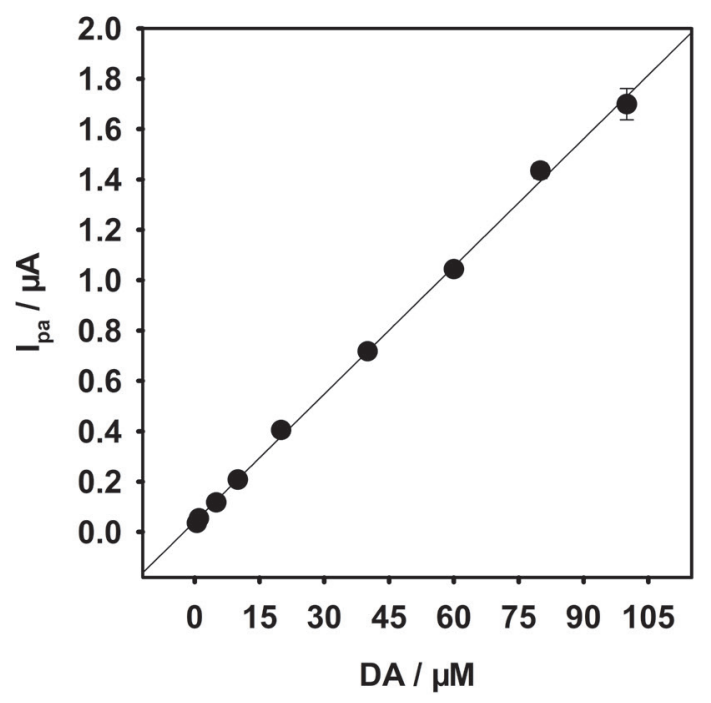

(b)

Fig. 9. (Color online) (a) LSV recorded at PEI-Fc/SFGO/GCE in $0.1 \mathrm{M} \mathrm{PBS}(\mathrm{pH} 7.0$, containing $0.1 \mathrm{M} \mathrm{KCl})$ containing different DA concentrations in the range of $0.5-100 \mu \mathrm{M}$ and (b) corresponding anodic peak currents as a function of the DA concentration; error bars are obtained using the standard error of the mean of three groups of data from three independent electrodes.

Table 1

Comparison of the as-prepared electrode for electrochemical sensing of DA with others.

\begin{tabular}{lcccc}
\hline Electrode & Linear range $(\mu \mathrm{M})$ & LOD $(\mu \mathrm{M})$ & Detection method & Reference \\
\hline $\mathrm{Graphene}^{\mathrm{a}} \mathrm{P}_{4} \mathrm{SAc} /{ }^{\mathrm{b}} \mathrm{CPE}$ & $5-100$ & 0.26 & $\mathrm{CV}$ & 30 \\
${ }^{\mathrm{c}} \mathrm{ZnO}-\mathrm{rGO} /{ }^{\mathrm{d}} \mathrm{Au}$ & $5-70$ & 0.167 & ${ }^{\mathrm{e}} \mathrm{DPV}$ & 31 \\
$\mathrm{MnO}_{2} / \mathrm{MWCNT} /{ }^{\mathrm{f}} \mathrm{EPGCE}$ & $0.5-30$ & 0.17 & $\mathrm{DPV}$ & 32 \\
$\mathrm{MWCNT}^{\mathrm{M}} \mathrm{COOH} /{ }^{\circ} \mathrm{Poly}(\mathrm{TB}) / \mathrm{GCE}$ & $1-30$ & 0.01039 & $\mathrm{DPV}$ & 33 \\
${ }^{\mathrm{h}} \mathrm{Cu}_{x} / \mathrm{ERGO} / \mathrm{Au}$ & $0.1-400$ & 0.012 & ${ }^{\mathrm{i}} \mathrm{AMP}$ & 34 \\
$\mathbf{P E I}-\mathrm{Fc} / \mathbf{S F G O} / \mathrm{GCE}$ & $\mathbf{0 . 5}-\mathbf{1 0 0}$ & $\mathbf{0 . 1}$ & LSV & Present work \\
\hline
\end{tabular}

${ }^{\mathrm{a}} \mathrm{P}_{4} \mathrm{SAc}$ : azobenzene-perylene diimide derivative

${ }^{\mathrm{b}} \mathrm{CPE}$ : carbon paste electrode

${ }^{\mathrm{C}} \mathrm{ZnO}$-rGO: zinc oxide ( $\left.\mathrm{ZnO}\right)$-decorated reduced graphene oxide ( $\left.\mathrm{ZnO}-\mathrm{rGO}\right)$ composites

${ }^{\mathrm{d}} \mathrm{Au}$ : gold electrode

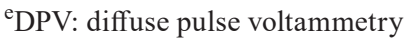

${ }^{f}$ EPGCE: electrochemically pretreated GCE

${ }^{g}$ Poly(TB): polymer of toluidine blue

${ }^{\mathrm{h}} \mathrm{CuxO} / \mathrm{ERGO}: \mathrm{Cu}_{2} \mathrm{O} / \mathrm{CuO} /$ electrochemically reduced graphene oxide

${ }^{\mathrm{i}} \mathrm{AMP}$ : amperometric technique

graphene $/ \mathrm{P}_{4} \mathrm{SAc}, \mathrm{ZnO}-\mathrm{rGO}, \mathrm{MnO}_{2} / \mathrm{MWCNT}$, and MWCNT-COOH/Poly(TB)-based DA sensors. ${ }^{(30-33)}$ Although the DA sensor based on $\mathrm{Cu}_{x} \mathrm{O} / \mathrm{ERGO} / \mathrm{Au}^{(34)}$ has an even wider linear range and a smaller LOD than those in the present work, oxygen gas must be introduced during the electrochemical deposition process for the fabrication of the $\mathrm{Cu}_{2} \mathrm{O} / \mathrm{CuO} / \mathrm{ERGO}$ nanocomposite, which makes the fabrication process inconvenient. Moreover, the stability of the DA sensor in the present work is higher. 


\subsection{Selectivity, reproducibility, repeatability, and stability}

The selectivity of the DA sensor is very important for its practical application since some biomolecules, especially, glucose, AA, and UA, co-exist with DA in biological samples. In addition, AA and UA exhibit similar oxidation potentials to DA. Therefore, the selectivity of the proposed DA sensor was studied using LSV by adding $500 \mu \mathrm{M}$ UA, $500 \mu \mathrm{M}$ glucose, and $50 \mu \mathrm{M}$ AA in $0.1 \mathrm{M}$ PBS containing $10 \mu \mathrm{M}$ DA. It can be seen from Fig. 10 that UA exhibited negligible interference with the electrooxidation peak current of DA and that glucose had no effect on the electrooxidation peak current of DA. After adding AA in $0.1 \mathrm{M}$ PBS containing $10 \mu \mathrm{M}$ DA, the anodic peak current increased by around $10 \%$. The greater interference of AA than of UA and glucose is attributed to the fact that AA has the closest oxidation potential to DA. To examine the reproducibility of the proposed DA sensor, five independent PEI-Fc/SFGO/GCE structures were fabricated and utilized to detect $50 \mu \mathrm{M}$ DA by CV in $0.1 \mathrm{M}$ PBS under the same conditions. The relative standard deviation (RSD) of the anodic peak currents was calculated to be $3 \%$, indicating the good reproducibility of the proposed DA sensor. The repeatability of PEI-Fc/SFGO/GCE was estimated by $\mathrm{CV}$ in $0.1 \mathrm{M}$ PBS containing $50 \mu \mathrm{M}$ DA for 10 successive cycles using the same $\mathrm{PEI}-\mathrm{Fc} / \mathrm{SFGO} / \mathrm{GCE}$. The anodic peak current $\left(I_{p a}\right)$ at the tenth cycle was still as high as $82 \%$ of that at the first cycle, and the RSD was evaluated to be 5\%, indicating the high repeatability and electrochemical stability of the as-prepared electrode. The stability was evaluated by recording the amperometric response of $50 \mu \mathrm{M}$ DA at a working potential of $0.25 \mathrm{~V}$ for 15 days. After each measurement, the DA sensor was stored at room temperature in PBS. As shown in Fig. 11, the amperometric response remained almost constant after 1 day of storage. However, there was an obvious decrease in amperometric response during 5 days of storage. The decrease is probably because some $\mathrm{Fc}^{+} / \mathrm{Fc}$ couples leaked or some SFGO detached from the electrode surface over

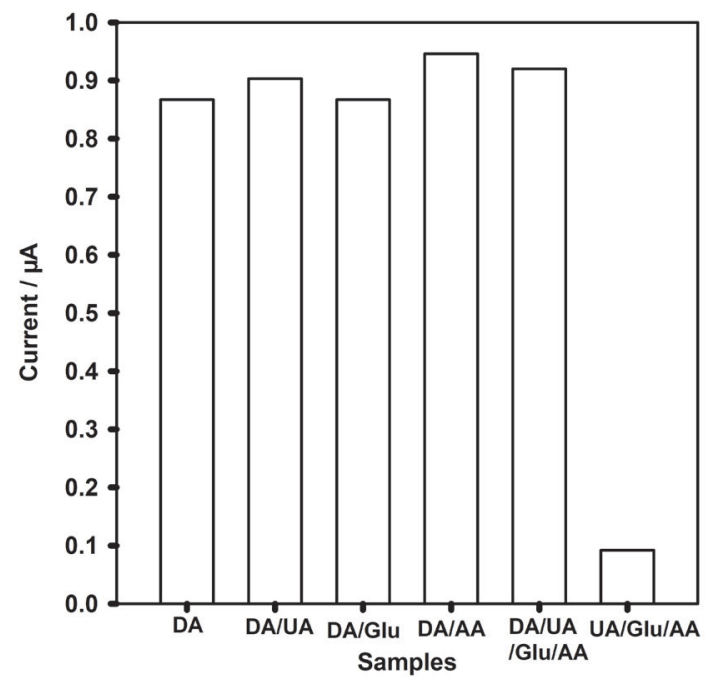

Fig. 10. Selectivity of the as-prepared PEI-Fc/SFGO/ GCE toward $10 \mu \mathrm{M}$ DA.

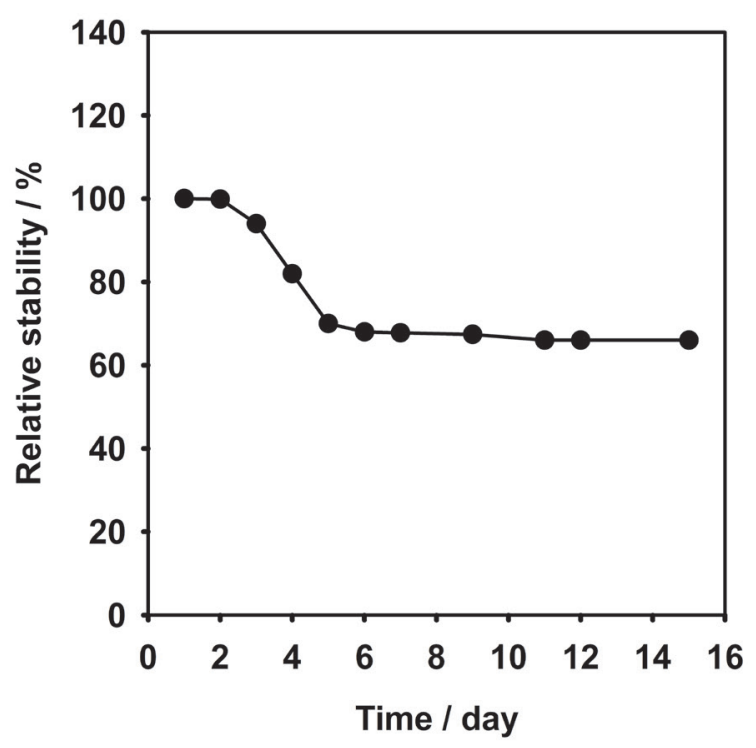

Fig. 11. Relative stability of the as-prepared PEI-Fc/ $\mathrm{SFGO} / \mathrm{GCE}$ toward $50 \mu \mathrm{M} \mathrm{DA}$. 
time. However, after 5 days, the amperometric response remained stable at around $70 \%$ of its initial response even up to 15 days of storage at room temperature, demonstrating the high stability of the as-prepared DA sensor. The high stability is probably due to the chemical stability of PEI-Fc and the mechanical stability of SFGO, which can protect the Fc groups from leakage out of the electrode surface.

\section{Conclusions}

An excellent PEI-Fc/SFGO composite was successfully prepared through a simple selfassembly method. SFGO provided a large specific surface area and high mechanical stability. PEI-Fc served as an effective electron transfer mediator and significantly facilitated the voltammetric response toward the electrooxidation of DA. As a result, the as-prepared DA sensor based on the PEI-Fc/SFGO composite modified electrode exhibited stable electrochemical activity, a wide linear range, high sensitivity, and a low detection limit for the detection of DA. In addition, the as-prepared DA sensor also exhibited high reproducibility and long-term stability, which demonstrated that the as-prepared PEI-Fc/SFGO composite is a potential electrode material for application in DA sensors.

\section{References}

1 Y. Li, Y. Gu, B. Zheng, L. Luo, C. Li, X. Yan, T. Zhang, N. Lu, and Z. Zhang: Talanta 162 (2017) 80. https://doi. org/10.1016/j.talanta.2016.10.016

2 Y. Dong, J. Liu, and J. Zheng: Colloids Surf. A 608 (2021) 125617. https://doi.org/10.1016/j.colsurfa.2020.125617

3 Q. Gong, H. Han, Y. Wang, C. Yao, H. Yang, and J. Qiao: New Carbon Mater. 35 (2010) 34. https://doi. org $110.1016 / \mathrm{S} 1872-5805(20) 60473-5$

4 A. Arroquia, I. Acosta, and M. Armada: Mater. Sci. Eng. C 109 (2020) 110602. https://doi.org/10.1016/j. msec.2019.110602

5 X. Ouyang, L. Luo, Y. Ding, B. Liu, D. Xu, and A. Huang: J. Electroanal. Chem. 748 (2015) 1. https://doi. org/10.1016/j.jelechem.2015.04.026

6 W. Zheng, M. Zhao, W. Liu, S. Yu, L. Niu, G. Li, H. Li, and W. Liu: J. Electroanal. Chem. 813 (2018) 75. https:// doi.org/10.1016/j.jelechem.2018.02.022

7 L. Yang, D. Liu, J. Huang, and T. You: Sens. Actuators, B 193 (2014) 166. https://doi.org/10.1016/j. snb.2013.11.104

8 P. Wiench, Z. González, R. Menéndez, B. Grzyb, and G. Gryglewicz: Sens. Actuators, B 257 (2018) 143. https://doi.org/10.1016/j.snb.2017.10.106

9 S. Wu, L. Xiao, Z. Du, H. Wana, Q. Yuan, and H. Ji: J. Electroanal. Chem. 804 (2017). 72. https://doi. org/10.1016/j.jelechem.2017.09.029

10 A. Ameri, M. Shakibaie, M. Khoobi, M. A. Faramarzi, A. Ameri, and H. Forootanfar: Appl. Biochem. Biotechnol. 191 (2020). 579. https://doi.org/10.1007/s12010-019-03180-1

11 J. Zhu, K. Zhang, K. Liu, and X. Shi: Constr. Build. Mater. 244 (2020) 118404. https://doi.org/10.1016/j. conbuildmat.2020.118404

12 D. Chen, H. Feng, and J. Li: J. Am. Chem. Soc. 112 (2012) 6027. https://doi.org/10.1021/cr300115g

13 S. Ayyaru and Y. Ahn: J. Membr. Sci. 525 (2017) 210. https://doi.org/10.1016/j.memsci.2016.10.048

14 G. Fadillah, W. P. Wicaksono, I. Fatimah, and T. A. Saleh: Microchem. J. 159 (2020) 105353. https://doi. org/10.1016/j.microc.2020.105353

15 F. Xiao, X. Yan, H. Li, C. Qian, S. Li, Y. Yu, C. Fan, M. Guo, J. Wang, S. Yang, Y. Xing, and H. Zhang: Sens. Actuators, B 288 (2019) 476. https://doi.org/10.1016/j.snb.2019.03.037

16 J. Qiu, W. Zhou, J. Guo, R. Wang, and R. Liang: Anal. Biochem. 385 (2009) 264. https://doi.org/10.1016/j. ab.2008.12.002

17 Q. Zhu, J. Bao, D. Huo, M. Yang, H. Wu, C. Hou, Y. Zhao, X. Luo, and H. Fa: J. Electroanal. Chem. 799 (2017) 459. https://doi.org/10.1016/j.jelechem.2017.07.004 
18 D. Zhu, D. Guo, L. Zhang, L. Tan, H. Pang, H. Ma, and M. Zhai: Sens. Actuators, B 281 (2018) 893. https://doi. org/10.1016/j.snb.2018.10.151

19 H. T. Zheng, H. Okada, S. Nojima, Sh. I. Suye, and T. Hori: Sci. Technol. Adv. Mater. 5 (2004) 371. https://doi. org/10.1016/j.snb.2018.10.151

20 N. Li, L. Zheng, F. Liao, H. T. Zheng, and S. I. Suye: Ionics 25 (2019) 2823. https://doi.org/10.1007/s11581-0182745-6

21 D. Sangamithirai, S. Munusamy, V. Narayanan, and A. Stephen: Surf. Interfaces 4 (2016) 27. https://doi. org/10.1016/j.surfin.2016.09.003

22 Z. Qiu, T. Yang, R. Gao, G. Jie, and W. Hou: J. Electroanal. Chem. 835 (2019) 123. https://doi.org/10.1016/j. jelechem.2019.01.040

23 C. Li, L. Xiao, Z. Jiang, X. Tian, L. Luo, W. Liu, Z. Xu, H. Yang, and Z. Jiang. Int. J. Hydrog. Energy 42 (2017) 16731. http://doi.org/10.1016/j.ijhydene.2017.05.126

24 M. Rouhani and A. Soleymanpour: Mater. Sci. Eng. C 117 (2020) 111287. https://doi.org/10.1016/j. msec. 2020.111287

25 M. S. Hosseini, M. Masteri-Farahani, and S. Shahsavarifar: J. Taiwan Inst. Chem. Eng. 102 (2019) 34. https:// doi.org/10.1016/j.jtice.2019.05.020

26 M. Chen, X. J. Wei, H. Qian, and G. W. Diao: Mater. Sci. Eng. C 31 (2011) 1271. https://doi.org/10.1016/j. msec.2011.04.002

27 L. A. Hernándeza, G. Riveros, F. Martí, D.M. González, M. C. Lopez, and M. León: Electrochim. Acta 240 (2017) 155. http://doi.org/10.1016/j.electacta.2017.04.076

28 G. Jiang, X. Gu, G. Jiang, T. Chen, W. Zhan, and S. Tian: Sens. Actuators, B 209 (2015) 122. https://doi. org/10.1016/j.snb.2014.11.109

29 W. Sroysee, S. Chairam, M. Amatatongchai, P. Jarujamrus, S. Tamuang, S. Pimmongkol, L. Chaicharoenwimolkul, and Ekasith Somsook: J. Saudi Chem. Soc. 22 (2018) 173. https://doi.org/10.1016/j. jscs.2016.02.003

30 T. Ö. Varol, B. Perk, O. Avci, O. Akpolat, Ö. Hakli, C. Xue, Q. Li, and Ü. Anik: Measurement 147 (2019) 106867. https://doi.org/10.1016/j.measurement.2019.106867

31 M. Cao, L. Zheng, Y. Gu, Y. Wang, H. Zhang, and X. Xua: Microchem. J. 159 (2020) 105465. https://doi. org/10.1016/j.microc.2020.105465

32 Y. Wang, L. Wang, and Q. Zhuang: J. Alloys Compd. 802 (2019) 326. https://doi.org/10.1016/j. jallcom.2019.06.124

33 V. N. Palakollu and R. Karpoormath: Synth. Met. 245 (2018) 87. https://doi.org/10.1016/j.synthmet.2018.08.012

34 H. Ö. Doğana, B. K. Urhan, E. Çepni, and M. Eryiğit: Microchem. J. 150 (2019) 104157. https://doi. org/10.1016/j.microc.2019.104157 